\title{
Postoperative nausea and vomiting in bariatric surgery in comparison to non-bariatric gastric surgery
}

\author{
Philipp Groene ${ }^{1}$, Jana Eisenlohr ${ }^{1}$, Catharina Zeuzem ${ }^{1}$, Sara Dudok ${ }^{1}$, Konrad Karcz², Klaus Hofmann-Kiefer ${ }^{1}$ \\ ${ }^{1}$ Department of Anaesthesiology, University Hospital, Ludwig Maximilian University, Munich, Germany \\ ${ }^{2}$ Department of General, Visceral and Transplantation Surgery, University Hospital, Ludwig Maximilian University, Munich, Germany
}

Videosurgery Miniinv 2019; 14 (1): 90-95

DOI: https://doi.org/10.5114/wiitm.2018.77629

\begin{abstract}
Introduction: Postoperative nausea and vomiting (PONV) are complications of general anesthesia. Patient-specific factors, type of surgery and a variety of drugs determine the frequency. Clinical experience shows nausea and vomiting to be very frequent in morbidly obese patients undergoing bariatric surgery.

Aim: To detect the onset and extent of nausea and vomiting in the group of morbidly obese patients undergoing laparoscopic bariatric surgery.

Material and methods: We conducted a retrospective data bank analysis (since 2004) of all patients with body mass index $>35 \mathrm{~kg} / \mathrm{m}^{2}$ undergoing laparoscopic bariatric surgery in comparison to patients with a body mass index $<35 \mathrm{~kg} / \mathrm{m}^{2}$ undergoing gastric surgery. Propensity score matching was applied to minimize bias effects. The frequency of postoperative nausea was defined as the primary outcome parameter.

Results: One hundred and thirty-eight patients were included. There was a significant difference between the morbidly obese group and the control group concerning the frequency of postoperative nausea (15.9\% vs. 55.1\%; $p<0.001)$. In patients receiving volatile anesthetics a significant difference between groups concerning frequency of PONV was not observed. Intravenous anesthetics were suitable to reduce PONV in the control group but not in the morbidly obese group (12.5\% vs. 56.8\%, $p<0.001)$. With given prophylaxis PONV events still occurred in $15.6 \%$ vs. $48.8 \%(p=0.003)$.

Conclusions: Morbidly obese patients undergoing laparoscopic bariatric surgery are at higher risk of suffering from PONV than non-morbidly obese patients. To reduce the PONV incidence in morbidly obese patients, further research, especially focusing on more efficient use of antiemetic drugs, seems to be necessary.
\end{abstract}

Key words: bariatric surgery, general anesthesia, postoperative nausea and vomiting, nausea, obese.

\section{Introduction}

Postoperative nausea and vomiting (PONV) are well-known complications of general anesthesia. In addition to various hypnotic drugs, as well as opioids (in a dose-dependent manner), patient-specific factors and the type of surgery determine the frequency of these complications. In several stud- ies, Apfel et al. were able to show that gender, postoperative use of opioids, smoking behavior, a history of PONV, a tendency to travel sickness, age, and the use of volatile anesthetics influence the risk of suffering from PONV $[1,2]$. In addition, there is evidence that younger patients, non-smokers and female patients tend to have postoperative nausea more often $[1,2]$.

\section{Address for correspondence}

Dr. Philipp Groene, Department of Anesthesiology, University Hospital of Munich, Ludwig-Maximilians-University, Marchioninistreet 15, 81377 Munich, Germany, phone: +49 17632972066, e-mail: Philipp.groene@med.uni-muenchen.de 
Abdominal surgery, especially if affecting the intestines or the stomach, can also lead to an increased incidence of PONV. In this field of surgery the fraction of morbidly obese patients is constantly increasing [1, 3].

After failure of conservative methods to lose body weight, bariatric surgery usually is the therapy of choice for morbidly obese patients. Bariatric surgery includes gastric bandage, sleeve gastrectomy and gastric bypass $[4,5]$. Due to their body weight and their increased body fat content, these patients usually receive very high quantities of hypnotics as well as opioids. Although the dosage of drugs used during general anesthesia should be based either on ideal body weight (IBW) or on lean body weight (LBW), the quantities of narcotics used in daily clinical practice are very high in the majority of institutions $[6,7]$.

Because of an increased content of body fat, the majority of morbidly obese patients receive volatile anesthetics to maintain general anesthesia [8]. In addition, remifentanil is frequently used in order to keep postoperative respiratory depression as low as possible. Postoperatively, bariatric patients appear to suffer from nausea and vomiting more frequently than normal weight or obese patients, but reliable scientific data are still lacking.

\section{Aim}

The aim of this retrospective cohort study was to detect the onset and extent of nausea and vomiting in the group of morbidly obese patients undergoing laparoscopic bariatric surgery. Postoperative nausea and vomiting was evaluated in the postoperative phase in the recovery room and compared to non-morbidly obese patients undergoing laparoscopic gastric surgery.

\section{Material and methods}

After approval of the Ethics Committee of the University of Munich, we accessed the department's patient documentation database for generating automatic anesthesia protocols (NarkoData, IMESO GmbH, Giessen, Germany) and its patient data management system (KAS, SAP Deutschland SE \& Co. KG, Walldorf, Germany). A retrospective evaluation of all bariatric patients of our institution scheduled for surgery was conducted from 01/2004 to $07 / 2016$. Informed consent was waived because patient data were irreversibly anonymized prior to extraction.

A body mass index $(\mathrm{BMI})>35 \mathrm{~kg} / \mathrm{m}^{2}$ (mentioned in the anesthesia protocol) and the corresponding procedure codes of laparoscopic bariatric surgery were entered as search criteria (morbidly obese group: OG) [9]. Patients with direct postoperative transfer to the intensive care unit (ICU) were excluded. Patients were labeled PONV "positive" either if the occurrence of nausea and/or vomiting was appropriately recorded by the recovery room's staff or if antiemetic drugs were administered in the recovery room. Antiemetic drugs taken into account were dimenhydrinate, ondansetron, tropisetron, granisetron, and droperidol. Non-morbidly obese patients (BMI $\left.<35 \mathrm{~kg} / \mathrm{m}^{2}\right)$ scheduled for gastric surgery during the same period were established as a control group (CG). To maintain comparability, the control group exclusively consisted of patients undergoing plain, laparoscopic gastric surgery (gastrectomies, fundoplications, partial gastrectomies), whereas patients suffering from gastric cancer or other malignant bowel diseases were not included. Similar to the OG, patients with direct transfer to the ICU were excluded as well as patients with epidural anesthesia.

The primary outcome parameter was the incidence of PONV, and the secondary outcome parameter was the frequency of antiemetic drug use. Further parameters examined were the duration of surgery, length of stay in the recovery room and opioid consumption. To facilitate comparability the various opioids administered are given in morphine equivalents according to their relative analgesic potency in comparison to morphine [10].

\section{Statistical analysis}

For all data analyses SPSS version 23 (IBM Corporation, Armonk, New York, USA), Microsoft Excel 2016 (Microsoft Corporation, Redmond, WA, USA) and XLSTAT (Addinsoft SARL, Paris, France) were used.

To account for potential confounders between study groups, each patient of group OG was matched to a normal weight control patient across 5 different variables: age, sex, ASA classification and dosage of postoperative opioids per kilogram LBW. In order to obtain the best matching results, i.e. to compare only patients who shared a maximum of similarities, the "nearest neighbor method" was applied 
and a strict $1: 1$ ratio was maintained with regard to the two study groups. Propensity score matching was done with XLSTAT and Microsoft Excel. The existence of a Gaussian distribution of data was evaluated using the Kolmogorov-Smirnov test; to test for equal variances the Levene test was applied. Data which were not normally distributed are displayed as median $+25^{\text {th }}$ and $75^{\text {th }}$ quartiles, otherwise they are given as mean \pm standard deviation of the mean. After matching, the Mann-Whitney $U$ test was used for group comparisons when data were not normally distributed; otherwise Student's $t$ test or Welch's test was applied. Associations regarding categorical and proportional demographic data and outcome variables were assessed using Pearson's $\chi^{2}$ test. For all comparisons, a value of $p<0.05$ was considered significant.

\section{Results}

Three hundred and ninety-five patients with a $\mathrm{BMI}>35 \mathrm{~kg} / \mathrm{m}^{2}$ who underwent a laparoscopic bariatric operation could be identified in the data system. On closer examination, 177 patients had to be excluded: In 75 cases patient's data were repeatedly listed, and 102 patients had to be excluded because of direct transfer to the ICU. Seven hundred and forty-one patients with a $\mathrm{BMI}<35 \mathrm{~kg} / \mathrm{m}^{2}$ and a gastric procedure could be identified for the CG, of which 664 had to be excluded: 226 because of multiple listing, 149 because of direct transfer to the ICU, 326 because of the kind of intervention (open surgery, extensive cancer surgery, multiple organ resection) and 40 because they had received an epidural anesthesia. Altogether, 295 patients meeting the criteria could be included in the matching process (BMI $>35 \mathrm{~kg} / \mathrm{m}^{2}: n=218 ; \mathrm{BMI}<35 \mathrm{~kg} / \mathrm{m}^{2}$ : $n=77$ ). Propensity score matching with a ratio $1: 1$ and the aforementioned criteria led to 138 patients being included in the study. Demographic data of the study population are given in Table I.

The compared cohorts differed in composition with respect to gender, age, body mass index and duration of surgery (Table I). Overall opioid consumption as well as opioid requirements in the recovery room did not differ significantly (Table I).

As demonstrated in Table II, there was a significant difference concerning the frequency of PONV

Table I. Demographic data

\begin{tabular}{|c|c|c|c|}
\hline \multirow[t]{2}{*}{ Parameter } & \multicolumn{2}{|c|}{$\mathrm{BMI}\left[\mathrm{kg} / \mathrm{m}^{2}\right]$} & \multirow[t]{2}{*}{$P$-value } \\
\hline & $<35$ & $>35$ & \\
\hline N & 69 & 69 & \\
\hline Sex, f/m [\%] & $44.9 / 55.1$ & $69.6 / 30.4$ & $0.003^{+}$ \\
\hline Age [years] & $59.3 \pm 13.9$ & $40.6 \pm 12.2$ & $<0.001^{*}$ \\
\hline BMI $\left[\mathrm{kg} / \mathrm{m}^{2}\right]$ & $27.6 \pm 4.1$ & $49.6 \pm 7.9$ & $<0.001^{*}$ \\
\hline Lean body weight $\left[\mathrm{kg} / \mathrm{m}^{2}\right]$ & $53.4 \pm 9.3$ & $72.3 \pm 13.0$ & $<0.001^{*}$ \\
\hline ASA classification [\%]: & & & $0.726^{+}$ \\
\hline 1 & 2.9 & 1.4 & \\
\hline 2 & 58.0 & 53.6 & \\
\hline 3 & 39.1 & 44.9 & \\
\hline Duration of surgery [min] & $130 \pm 51.4$ & $105.7 \pm 40.2$ & $<0.001^{*}$ \\
\hline Time in recovery room [min] & $121.0(95.0-160.0)$ & $115.0(86.0-182.5)$ & $0.895^{\#}$ \\
\hline Opioid per kg body weight in mg morphine & $0.86(0.70-1.32)$ & $0.78(0.49-2.46)$ & $0.459^{\star}$ \\
\hline Opioid per kg LBW in mg morphine & $1.33(1.06-1.97)$ & $1.58(0.99-4.82)$ & $0.213^{*}$ \\
\hline Opioid per kg LBW in recovery room in mg morphine & $0.00(0.0-0.10)$ & $0.04(0.0-0.09)$ & $0.683^{\S}$ \\
\hline
\end{tabular}

Data are given as mean \pm standard deviation of the mean, median $+25^{\text {th }}$ and $75^{\text {th }}$ quartiles and as proportions, respectively, "significant difference between CG and OG; Mann-Whitney U test, ${ }^{*}$-test, ${ }^{+} \chi^{2}$ test, ${ }^{5}$ Welch test. 
between the CG and OG (15.9\% vs. $55.1 \%$; $p<$ 0.001). A gender-related subdivision of the study groups did not provide results different from those for the whole cohort. We observed a significant difference in the frequency of PONV between the CG and OG when sex was female and when it was male ( $p=0.022$ (male); $p<0.001$ (female); Table II).

However, as demonstrated in Table III, there was no significant difference between the CG and OG concerning the frequency of PONV when volatile anesthetics were used to maintain anesthesia (29.4\% vs. $48.5 \%$; $p=0.196)$. In contrast, the administration of intravenous anesthetics (propofol) led to a significant difference in the incidence of PONV between groups (Table III; $12.5 \%$ vs. 56.8\%; $p<0.001)$.

In bariatric patients who received a PONV prophylaxis, PONV events occurred in $48.8 \%$ of the subjects. In the non-bariatric cohort, this was the case in only $15.6 \%(p=0.003)$. Prophylaxis was given in $46.6 \%$ of the cases in the CG and $59.4 \%$ in the OG $(p=0.125)$. The type of prophylaxis differed. Dexamethasone (4 mg) and dexamethasone (4 mg) + ondansetron (4 mg) were the most common forms of prophylaxis.

Sleeve gastrectomy was the most frequent surgery in $O G$ and fundoplication the most frequent procedure in the CG. Other types of surgery were gastric bypass and gastric banding in $O G$ and partial gastrectomy and gastrectomy in the CG (data not shown). Ulcer was the most common reason for gastrectomy or partial gastrectomy in the CG.

\section{Discussion}

The current study compared the frequency of postoperative nausea in morbidly obese and normal weight to mildly obese patients in 138 cases and observed significant differences concerning the study population. The frequency of PONV in the OG we observed in the current study was comparable to other investigations $[11,12]$. In addition, the occurrence of PONV was more
Table II. Frequency of PONV

\begin{tabular}{|lccc|}
\hline PONV & Control group & Obese group & $P$-value \\
\hline Yes, $n(\%):$ & $11(15.9)$ & $38(55.1)$ & $<0.001^{\star}$ \\
\hline Male & $6(15.8)$ & $9(42.9)$ & $0.022^{*}$ \\
\hline Female & $5(16.1)$ & $29(60.4)$ & $<0.001^{\star}$ \\
\hline
\end{tabular}

Data are given as quantities and proportions: *significant difference between $C G$ and $O G, \chi^{2}$ test.

frequent in female subjects and, thus, corresponds to the results reported by Apfel et al. [1, 13]. Gender-related subgroup-analyses also showed significant differences between the CG and OG, which were well in line with the overall PONV rates of this study.

Postoperative nausea and vomiting are major side effects of general anesthesia. They not only lead to reduced well-being, but might also provoke serious adverse events, in addition to prolonged hospital stays and increased treatment costs [14, 15]. For example, increased abdominal and thoracic pressure caused by vomiting can cause a rupture of sutures and bowel anastomoses and, thus, can lead to bleeding and anastomotic leakage.

There are already clearly identified risk factors for PONV, including female gender, non-smoking status, positive PONV history, and travel sickness $[1,14]$. In laparoscopic abdominal surgery, postoperative nausea is particularly common [1]. According to the literature the frequency of PONV can be up to 70\% after laparoscopic procedures [16].

However, in the field of bariatric surgery, up to now, there is only a small number of studies focusing on PONV rates and their cause. Mendes et al. and Moussaa et al. reported on PONV-incidences up to $79 \%$ in bariatric patients $[11,12]$. In a study published by Bataille et al., who examined PONV rates when using total intravenous anaesthesia with BIS monitoring, PONV frequency was up to 54\% [17]. Subgroups analyses concerning the type of narcotics administered, gender or type of surgery were not included in any of these investigations.

Table III. Frequency of PONV when using volatile or intravenous anesthetics

\begin{tabular}{|lccccc|}
\hline PONV & \multicolumn{3}{c|}{ Using volatile anesthetics } & \multicolumn{2}{c|}{ Using intravenous anesthetics } \\
\cline { 2 - 5 } & Control group & Obese group & $P$-value & Control group & Obese group \\
\hline Yes, $n(\%)$ & $5(29.4)$ & $16(48.5)$ & 0.196 & $7(12.5)$ & $25(56.8)$ \\
\hline No, $n(\%)$ & $12(70.6)$ & $17(51.5)$ & & $49(87.5)$ & $19(43.2)$ \\
\hline
\end{tabular}

Data are given as quantities and proportions, $\chi^{2}$ test (Pearson). 
The analyses of the anesthetics used provided interesting results: Although overall PONV frequency differed significantly between groups OG and CG, we did not observe significant intergroup differences when volatile anesthetics were administered. This is remarkable because Apfel et al. demonstrated the administration of volatile anesthetics to be a risk factor for PONV [1]. One can speculate that the noteworthy increase in PONV frequency under general anesthesia with volatile anesthetics in patients undergoing gastric surgery (morbidly obese as well as normal weight) masks the differences caused by the differences in body weight. But this fact can also be due to underpowered small groups and could be of interest in further research on this topic. When intravenous anesthetics were used, PONV rates in the control group were as low as expected [18]. Howev$\mathrm{er}$, in the obese group, intravenous anesthetics were not able to prevent or reduce nausea and vomiting. The PONV rate remained at $56 \%$, which is in contradiction to the risk factors known up to now [1].

Type of surgery could have had an effect on PONV rates. Fundoplication was the most common surgery in the CG. Passage problems can occur after surgery and may result in nausea and vomiting. This can also happen with gastric banding, which was one of the procedures in the OG. Gastrectomies and partial gastrectomies were included in the CG. In these cases, physiology should be the same as after bariatric surgery (e.g. sleeve gastrectomy). Therefore, groups are comparable concerning types of surgery.

Concerning therapy of PONV, previous studies on severely overweight patients showed that combinations of antiemetic drugs are more efficient to successfully treat nausea and vomiting; in some studies, even a combination of two drugs seemed not to be satisfactory $[11,12,15,17,19]$. This was also valid if propofol instead of volatile anesthetics was used to maintain anesthesia [17]. In the current study, which explicitly did not focus on this topic, many different regimes to prevent and treat PONV were applied, which clearly reflects the lack of validated concepts how to deal with PONV in bariatric surgery. In this context, it is interesting that, in addition to the risk factors identified by Apfel et al., hormones and transmitters may play a significant role as catalysts for PONV in morbidly obese patients during bariatric surgery [17]. One reason for a failure of antiemetics could be release of 5-hydroxytryptamine. This hormone is produced and stored in the enterochromaffin cells of the gastrointestinal tract and in the central nervous system. The release of 5-hydroxytryptamine is associated with nausea and vomiting. Gastric sleeve resection as well as gastric bypass - as a surgical trauma - can provoke enhanced release of this hormone and, as a consequence, increase the frequency of PONV in morbidly obese subjects. This effect seems to play a more substantial role in obese than in non-morbidly obese patients [17]. Thus, investigating this "hormonal approach" may be worthwhile with regard to improved PONV therapy especially in this group of patients.

Although propensity score matching was used to align the two cohorts, there are still some limitations. Similarly to other retrospective investigations, we were not able to completely rule out a certain personal bias especially concerning the administration of antiemetics, which served as a main inclusion criterion. However, this limitation concerned both study groups and should not have influenced group comparisons. In addition, the study was designed to examine the incidence but not the severity of PONV in patients with upper abdominal surgery. In group OG the proportion of women was significantly higher than in group CG. The reason for this phenomenon is unclear. However, one can speculate that women more frequently tend to undergo "cosmetic surgery" than men. Because female gender - like obesity - is a risk factor for PONV, this may have influenced the results. Data related to other established risk factors of PONV, such as smoking or previous occurrence of PONV, were not examined.

\section{Conclusions}

Morbidly obese patients undergoing laparoscopic bariatric surgery are at higher risk of suffering from PONV. To reduce the PONV incidence in obese patients, further research, especially focusing on improvements in anesthesiologic management and on more efficient use of antiemetic drugs, seems to be necessary.

\section{Conflict of interest}

The authors declare no conflict of interest.

\section{References}

1. Apfel CC, Heidrich FM, Jukar-Rao S, et al. Evidence-based analysis of risk factors for postoperative nausea and vomiting. $\mathrm{Br}$ J Anaesth 2012; 109: 742-53. 
2. Apfel CC, Laara E, Koivuranta M, et al. A simplified risk score for predicting postoperative nausea and vomiting: conclusions from cross-validations between two centers. Anesthesiology 1999; 91: 693-700.

3. Le TP, Gan TJ. Update on the management of postoperative nausea and vomiting and postdischarge nausea and vomiting in ambulatory surgery. Anesthesiol Clin 2010; 28: 225-49.

4. Major P, Janik MR, Wysocki M, et al. Comparison of circular- and linear-stapled gastrojejunostomy in laparoscopic Roux-en-Y gastric bypass: a multicenter study. Videosurgery Miniinv 2017; 12: 140-6.

5. Jędrzejewski E, Liszka M, Maciejewski M, et al. Age is not associated with increased surgical complications in patients after laparoscopic sleeve gastrectomy. Videosurgery Miniinv 2018; 13: 82-7.

6. Dong D, Peng X, Liu J, et al. Morbid obesity alters both pharmacokinetics and pharmacodynamics of propofol: dosing recommendation for anesthesia induction. Drug Metabol Disposition 2016; 44: 1579-83.

7. Li JY, Zou QR, Peng XM. Pharmacokinetics of a cisatracurium dose according to fat-free mass for anesthesia induction in morbidly obese patients. J South Med Univ 2016; 36: 1396-400.

8. Bolkenius D, Dumps C, Halbeck E. Drugs for intravenous induction of anesthesia: propofol. Anaesthesist 2018; 67: 147-62.

9. Krollner B, Krollner M. ICD-10-GM-2017 Code [Internet]. icd-code. de. 2017 [cited 2017 Nov 18]. Available from: http://www.icdcode.de/suche/ops/recherche.html?sp=0\&sp=Sgastrektomie

10. Feldman PL, James MK, Brackeen MF, et al. Design, synthesis, and pharmacological evaluation of ultrashort- to long-acting opioid analgetics. J Med Chem 1991; 34: 2202-8.

11. Mendes MN, Monteiro RES, Martins FA. Prophylaxis of postoperative nausea and vomiting in morbidly obese patients undergoing laparoscopic gastroplasties: a comparative study among three methods. Rev Bras Anestesiol 2009; 59: 570-6.

12. Moussa AA, Oregan PJ. Prevention of postoperative nausea and vomiting in patients undergoing laparoscopic bariatric surgery: granisetron alone vs granisetron combined with dexamethasone/droperidol. Middle East J Anaesthesiol 2007; 19: 357-67.

13. Apfel CC, Kranke P, Piper S, et al. Nausea and vomiting in the postoperative phase. Expert- and evidence-based recommendations for prophylaxis and therapy. Anaesthesist 2007; 56: 1170-80.

14. Maitra S, Som A, Baidya DK, Bhattacharjee S. Comparison of ondansetron and dexamethasone for prophylaxis of postoperative nausea and vomiting in patients undergoing laparoscopic surgeries: a meta-analysis of randomized controlled trials. Anesthesiol Res Pract 2016; 2016: 7089454.

15. Leksowski K, Peryga P, Szyca R. Ondansetron, metoclopramid, dexamethason, and their combinations compared for the prevention of postoperative nausea and vomiting in patients undergoing laparoscopic cholecystectomy: a prospective randomized study. Surg Endosc 2006; 20: 878-82.

16. Thune A, Appelgren L, Haglind E. Prevention of postoperative nausea and vomiting after laparoscopic cholecystectomy. A prospective randomized study of metoclopramide and transdermal hyoscine. Eur J Surg 1995; 161: 265-8.

17. Bataille A, Letourneulx JF, Charmeau A, et al. Impact of a prophylactic combination of dexamethasone-ondansetron on postoperative nausea and vomiting in obese adult patients undergoing laparoscopic sleeve gastrectomy during closed-loop propofol-remifentanil anaesthesia: a randomised double-blind placebo-controlled study. Eur J Anaesthesiol 2016; 33: 898-905.

18. Jost U, Dorsing C, Jahr C, et al. Propofol and postoperative nausea and/or vomiting. Anaesthesist 1997; 46: 776-82.

19. Benevides ML, Oliveira SS, de Aguilar-Nascimento JE. The combination of haloperidol, dexamethasone, and ondansetron for prevention of postoperative nausea and vomiting in laparoscopic sleeve gastrectomy: a randomized double-blind trial. Obes Surg 2013; 23: 1389-96.

Received: 21.04.2018, accepted: 13.07.2018. 